\title{
Ocular involvement in systemic lupus erythematosus patients: a paradigm shift based on the experience of a tertiary referral center
}

\author{
A Dias-Santos ${ }^{1,2,3}$ D, J Tavares Ferreira ${ }^{1,2,3}$, S Pinheiro ${ }^{4}$, JP Cunha ${ }^{1,3}$, M Alves ${ }^{5}$, AL Papoila ${ }^{3,5,6}$, \\ M Francisca Moraes-Fontes ${ }^{3,7,8}$ (D) and R Proença ${ }^{9,10}$ \\ ${ }^{1}$ Department of Ophthalmology, Centro Hospitalar e Universitário de Lisboa Central, Lisbon, Portugal; ${ }^{2}$ Department of Ophthalmology, Hospital \\ CUF Descobertas, Lisbon, Portugal; ${ }^{3}$ NOVA Medical School, Universidade NOVA de Lisboa, Lisbon, Portugal; ${ }^{4}$ Autoimmune Disease Unit, \\ Unidade de Doenças Auto-imunes/Serviço Medicina 3, Hospital de Santo António dos Capuchos, Centro Hospitalar e Universitário de Lisboa \\ Central, Lisbon, Portugal; ${ }^{5}$ Epidemiology and Statistics Unit, Research Center, Centro Hospitalar e Universitário de Lisboa Central, Lisbon, \\ Portugal; ${ }^{6}$ CEAUL (Center of Statistics and Applications), Lisbon University, Lisbon, Portugal; ${ }^{7}$ Autoimmune Disease Unit, Unidade de Doenças \\ Auto-imunes/Serviço de Medicina 7.2, Hospital Curry Cabral, Centro Hospitalar e Universitário de Lisboa Central, Lisbon, Portugal; ${ }^{8}$ Instituto \\ Gulbenkian de Ciência, Oeiras, Portugal; ${ }^{9}$ Department of Ophthalmology, Centro Hospitalar e Universitário de Coimbra, Coimbra, Portugal; and \\ ${ }^{10}$ Faculty of Medicine, University of Coimbra, Coimbra, Portugal
}

\begin{abstract}
Objective: To evaluate ocular involvement in a cohort of systemic lupus erythematosus (SLE) patients of a tertiary referral center and to compare the results with the existing literature. Methods: Patients underwent a complete ophthalmological evaluation, including visual acuity, slit-lamp examination, fluorescein staining, Schirmer-I test, Goldmann applanation tonometry, fundoscopy, 10-2 automated threshold visual fields, fundus autofluorescence and spectral-domain optical coherence tomography to screen for hydroxychloroquine (HCQ) macular toxicity. Results: A total of 161 patients (16 men and 145 women) were enrolled in this study. The mean age was 47.6 years and the mean disease duration was 11.5 years. Fifty patients $(31.1 \%)$ had at least one ocular manifestation of SLE. The most frequent manifestation was dry eye syndrome $(12.4 \%)$, immediately followed by cataracts $(11.2 \%)$ and HCQ macular toxicity (11.2\%). Among patients with HCQ maculopathy, two presented with an atypical spectral-domain optical coherence tomography pattern. Five patients $(3.1 \%)$ presented with glaucoma, two patients $(1.2 \%)$ presented with SLE retinopathy while only one presented with lupus choroidopathy $(0.6 \%)$. Conclusions: Compared with previous studies, we conclude there has been a significant reduction in disease-related ocular complications, particularly those associated with poor systemic disease control. On the other hand, drug and age-related complications are assuming a prominent role in the ophthalmic care of these patients. Lupus (2020) 29, 283-289.
\end{abstract}

Key words: Glucocorticoids; hydroxychloroquine; maculopathy; ocular manifestations; systemic lupus erythematosus

\section{Introduction}

Systemic lupus erythematosus (SLE) is a chronic autoimmune connective tissue disease that can involve any organ system. Ocular involvement occurs in approximately one third of SLE patients and virtually any ocular structure can be affected. Keratoconjunctivitis sicca is the most frequent ophthalmic complication, affecting nearly $33 \%$,

Correspondence to: Arnaldo Dias-Santos, Serviço de Oftalmologia, Hospital de Santo António dos Capuchos, Alameda de Santo António dos Capuchos, Lisboa, 1169-050, Portugal.

Email: arnaldomiguelsantos@gmail.com

Received 23 October 2019; accepted 8 January 2020 frequently associated with secondary Sjogren's syndrome. ${ }^{1}$ Posterior segment involvement, namely lupus retinopathy, choroidopathy and optic neuropathy is particularly concerning given their potential for a devastating impact on visual prognosis and their relation with poor systemic disease control. ${ }^{2}$ According to most reports, lupus retinopathy is the second most common ocular manifestation with an incidence that may reach $29 \% .^{3}$ However, over the last few years we have been entering a new era in the treatment of SLE. Biologic agents have entered the therapeutic armamentarium, and antimalarials, particularly hydroxychloroquine (HCQ), have been introduced as long-term maintenance therapy to almost every patient early in the course 
Ocular involvement in SLE A Dias-Santos et al

of the disease. ${ }^{4}$ Consequently, there has been a significant improvement in systemic disease control, a reduction in lupus flares, with a marked decrease in morbidity and mortality. As a result of these advances, changes in ophthalmic manifestations are expected, with a reduction in disease-related complications and an increase in age-related complications as well as an increase in drug-related ocular toxicity.

The objective of this study was to evaluate ocular involvement in a cohort of SLE patients of a tertiary referral center and to compare the results with the existing literature.

\section{Materials and methods}

This prospective study was conducted at the Ophthalmology Department of Central Lisbon Hospital and University Center between July 2017 and July 2019. SLE patients were sent for ophthalmological evaluation by the Autoimmune Disease Unit of the hospital, which is a tertiary referral center for SLE. All patients fulfilled the updated American College of Rheumatology criteria for the diagnosis of SLE. ${ }^{5}$ Patients underwent a complete ophthalmological evaluation that included best corrected visual acuity, slit-lamp examination, Goldmann applanation tonometry, Schirmer-I test with topical anesthesia, fluorescein staining and fundoscopy under pharmacological mydriasis. In patients currently or previously medicated with HCQ, 10-2 automated threshold visual fields, fundus autofluorescence (FAF) and spectraldomain optical coherence tomography (SD-OCT) were performed to screen for macular toxicity. To those in whom macular toxicity was suspected based on the above-mentioned examinations, multifocal electroretinogram (mfERG) was also performed. Toxicity diagnosis required an abnormal visual field examination confirmed by at least one of the above-mentioned objective examinations, according to the American Academy of Ophthalmology guidelines. ${ }^{6}$ Patients were then divided in two groups according to the presence or absence of HCQ maculopathy and a comparison of the clinical characteristics between groups was performed. This study was approved by our Institutional Ethics Committee and written informed consent was obtained from all participants. The tenets of the Declaration of Helsinki were followed.

\section{Statistical analysis}

Qualitative data were presented as frequencies (percentages) and quantitative data as means (standard deviation). Prevalence was presented with 95\% confidence intervals. Normality of the variables was verified using the Shapiro-Wilk test and the difference between groups was tested by chisquare and the Student's $t$-test. A level of significance $\alpha=0.05$ was considered. Data were analyzed using the Statistical Package for the Social Sciences for Windows (IBM Corp. Released 2013. IBM SPSS Statistics for Windows, Version 22.0. Armonk, NY: IBM Corp.) and OpenEpi (Dean AG, Sullivan KM, Soe MM. OpenEpi: Open Source Epidemiologic Statistics for Public Health, Version www.OpenEpi.com, updated 06/04/2013, accessed 22/10/2019).

\section{Results}

A total of 161 patients (16 [9.9\%] men and 145 [90.1\%] women) were enrolled in this study. The mean age was 47.6 (standard deviation: 13.4) years and the mean disease duration was 11.5 (standard deviation: 5.1) years. Sixty-six patients $(41.0 \%)$ had at least one sign of ophthalmic disease; however, when we considered only those manifestations directly related to the disease or its therapy (dry eye syndrome, cataracts, HCQ toxicity, glaucoma, lupus retinopathy or choroidopathy and uveitis), this number dropped to 50 patients $(31.1 \%)$. Among ophthalmic manifestations, the most frequent was dry eye syndrome, followed by cataracts or previous cataract surgery (Table 1). Regarding medication with HCQ, 130 (80.7\%) patients were currently medicated with this drug, $27(16.8 \%)$ had been treated with it previously but had discontinued and four $(2.5 \%)$ patients were treatment naïve at the time of the evaluation. Eighteen $(11.2 \%)$ patients met the criteria for HCQ macular toxicity. Among the patients with HCQ maculopathy, one patient had classic bull's eye maculopathy (Figure 1), one had cystoid macular degeneration (Figure 2) and one had pigment epithelial detachment, which spontaneously resolved 6 months after discontinuing the drug (Figure 3). The remaining 15 patients had classic parafoveal photoreceptor layer thinning with corresponding visual field defects (Figure 4). Only the patient with bull's eye maculopathy complained of decreased visual acuity, the remaining were asymptomatic. 
In Table 2 we compare SLE patients with and without HCQ maculopathy. Patients with macular toxicity were significantly older than those without this complication $(p=0.014)$. Additionally, there was a statistically significant difference between these two groups regarding HCQ cumulative dosage $(p<0.001)$ and treatment time $(p<0.001)$, which were higher in the group with macular toxicity. HCQ was discontinued in all patients meeting the

Table 1 Prevalence of ocular disease in SLE patients

\begin{tabular}{lll}
\hline Ocular abnormalities & Frequency & $\begin{array}{l}\%(95 \% \text { confidence } \\
\text { interval) }\end{array}$ \\
\hline Dry eye syndrome & 20 & $12.4(8.2-18.4)$ \\
Cataract or previous cataract surgery & 18 & $11.2(7.2-17.0)$ \\
Hydroxychloroquine retinopathy & 18 & $11.2(7.2-17.0)$ \\
High myopia & 7 & $4.3(2.1-8.7)$ \\
Glaucoma & 5 & $3.1(1.3-7.1)$ \\
Amblyopia & 4 & $2.5(1.0-6.2)$ \\
Strabismus & 3 & $1.9(0.6-5.3)$ \\
Lupus retinopathy & 2 & $1.2(0.3-4.4)$ \\
Macular epiretinal membrane & 2 & $1.2(0.3-4.4)$ \\
Lupus choroidopathy & 1 & $0.6(0.1-3.4)$ \\
Pterygium & 1 & $0.6(0.1-3.4)$ \\
Conjunctival intraepithelial neoplasia & 1 & $0.6(0.1-3.4)$ \\
Anterior uveitis & 1 & $0.6(0.1-3.4)$ \\
Fuchs endothelial dystrophy & 1 & $0.6(0.1-3.4)$ \\
Bilateral Coloboma & 1 & $0.6(0.1-3.4)$ \\
Choroidal nevus & 1 & $0.6(0.1-3.4)$ \\
\hline
\end{tabular}

criteria of macular toxicity. Good systemic disease control allowed, in most cases, a simple withdrawal of HCQ. In only two cases was azathioprine dosage increased in order to solve disease exacerbation.

\section{Discussion}

In this study, which included 161 SLE patients, disease-related ocular manifestations were present in $31.1 \%$ of patients, a proportion that is in line with previous studies. ${ }^{7,8}$ However, the relative frequency of each ophthalmic manifestation has changed compared with past studies. There has been an increase in drug and age-related manifestations and a decrease in disease-related ocular complications, particularly those directly linked to systemic disease activity. To our knowledge, this is the first epidemiologic study on ophthalmic manifestations of SLE performed in Portugal.

Dry eye syndrome was the most common form of ocular involvement in this cohort, with a prevalence of $12.4 \%$, which is in line with previous reports. 9,7 However, the proportion of patients with lupus retinopathy has significantly decreased compared with past studies. It was previously reported as the second most common ocular complication, with a prevalence of $7-29 \% .^{10}$ In our study, only $1.2 \%$ of patients presented with this

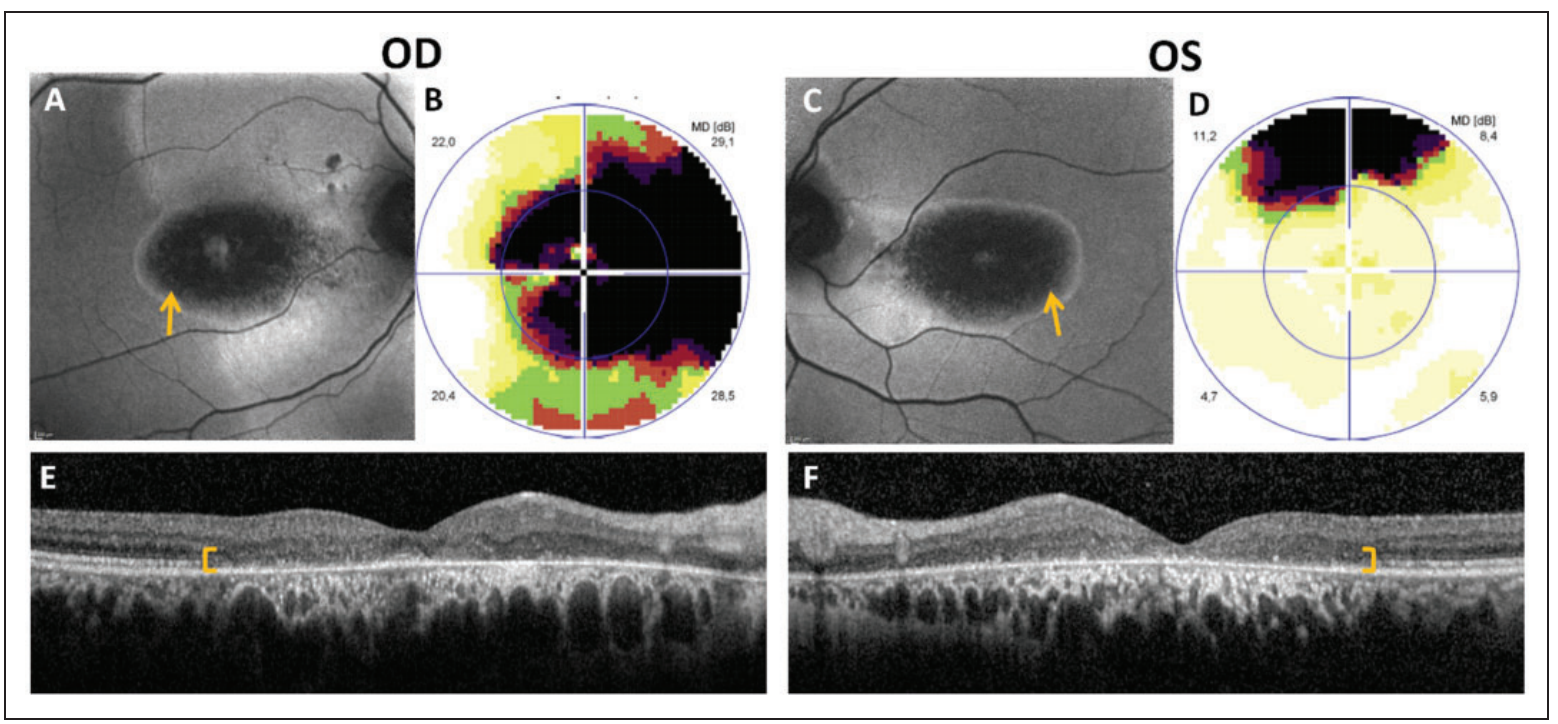

Figure 1 Fundus autofluorescence (A and C), macular visual field results (B and D) and spectral-domain optical coherence tomography (E and F) of a 42-year-old male patient with bull's eye maculopathy. Yellow arrows point out the central ring of reduced autofluorescence, corresponding to advanced retinal pigment epithelium loss. The yellow marks on optical coherence tomography images highlight the central area of outer retinal damage, with marked disruption of photoreceptors and retinal pigment epithelium layers. 


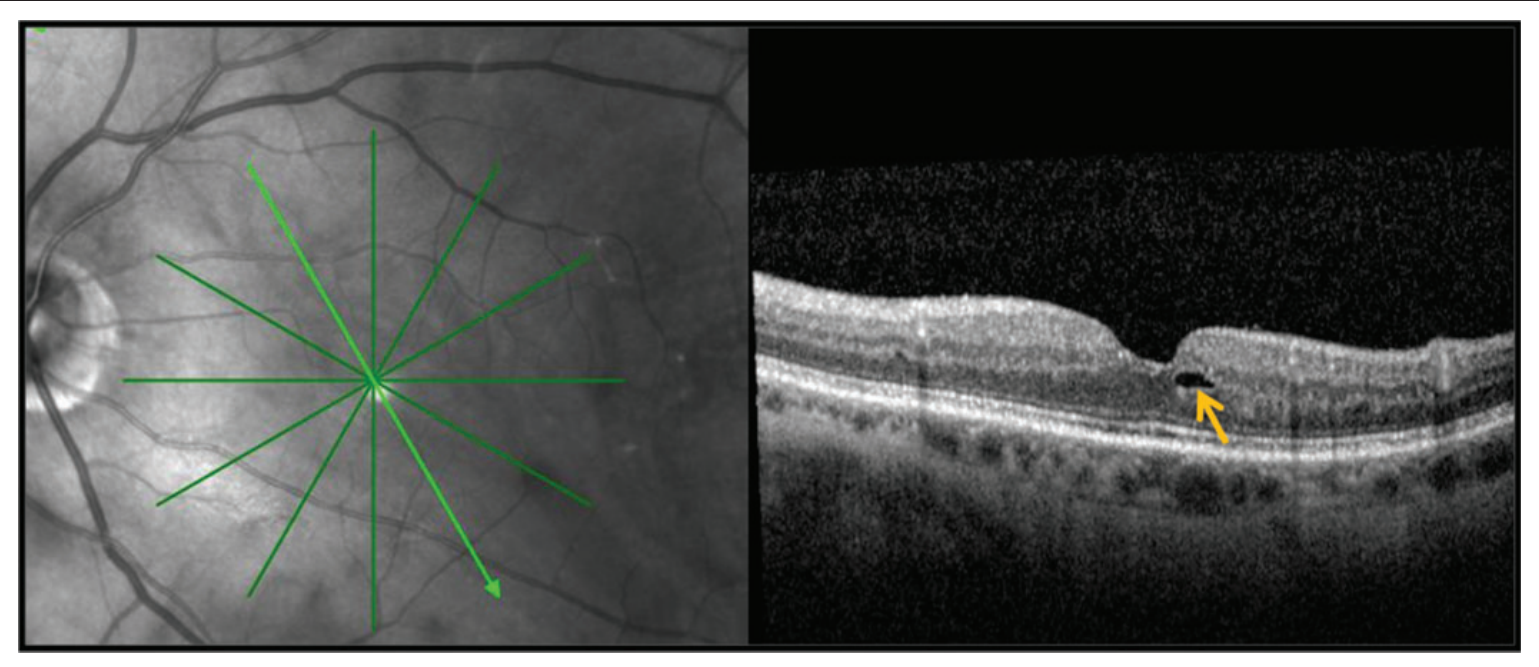

Figure 2 Spectral-domain optical coherence tomography of a 69-year-old patient with unilateral macular cystoid degeneration (yellow arrow).

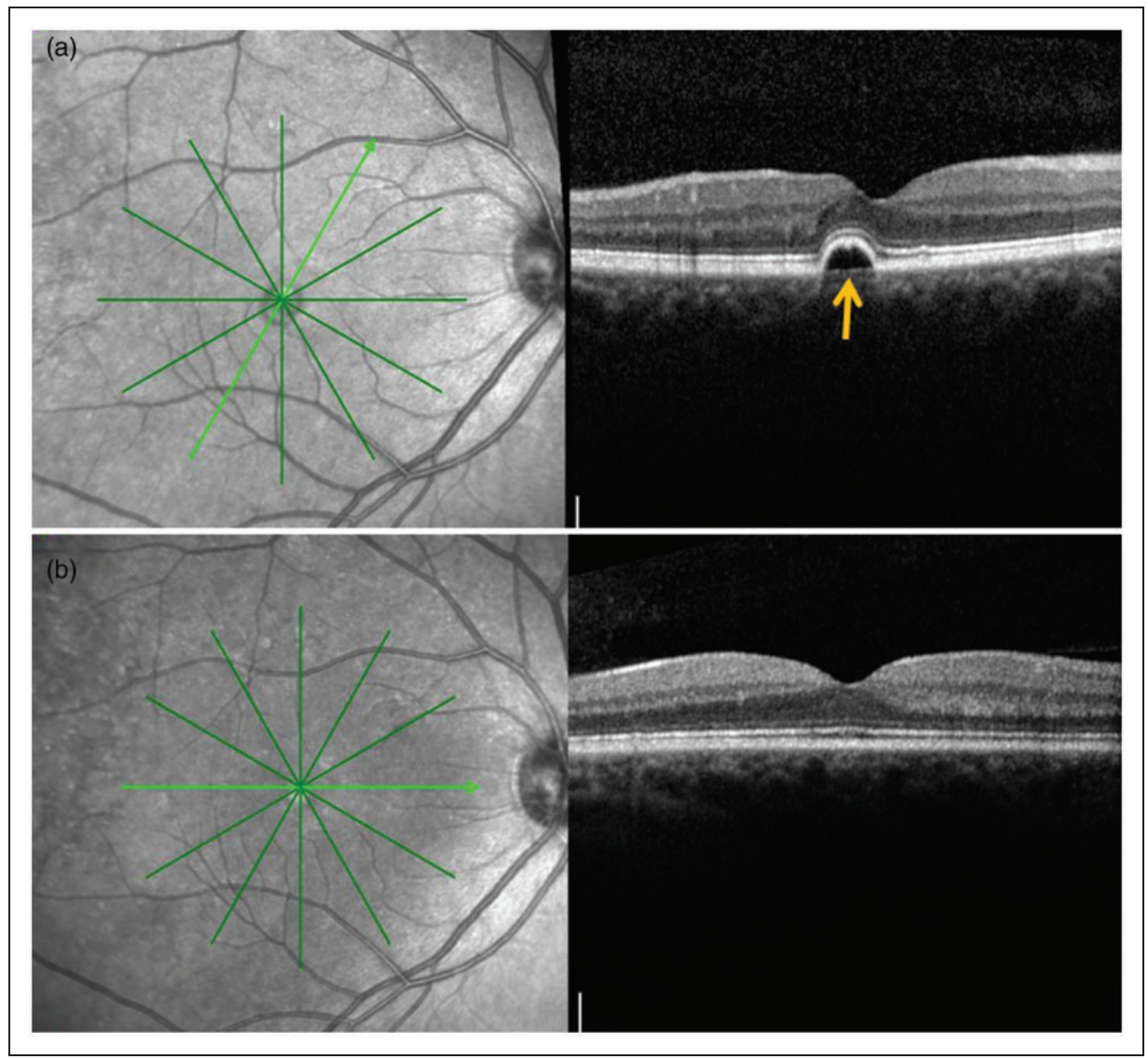

Figure 3 Spectral-domain optical coherence tomography of a 48-year-old female on hydroxychloroquine therapy for 20 years showing unilateral pigment epithelial detachment (a) (yellow arrow). Six months after stopping the drug, the pigment epithelial detachment resolved spontaneously (b). 

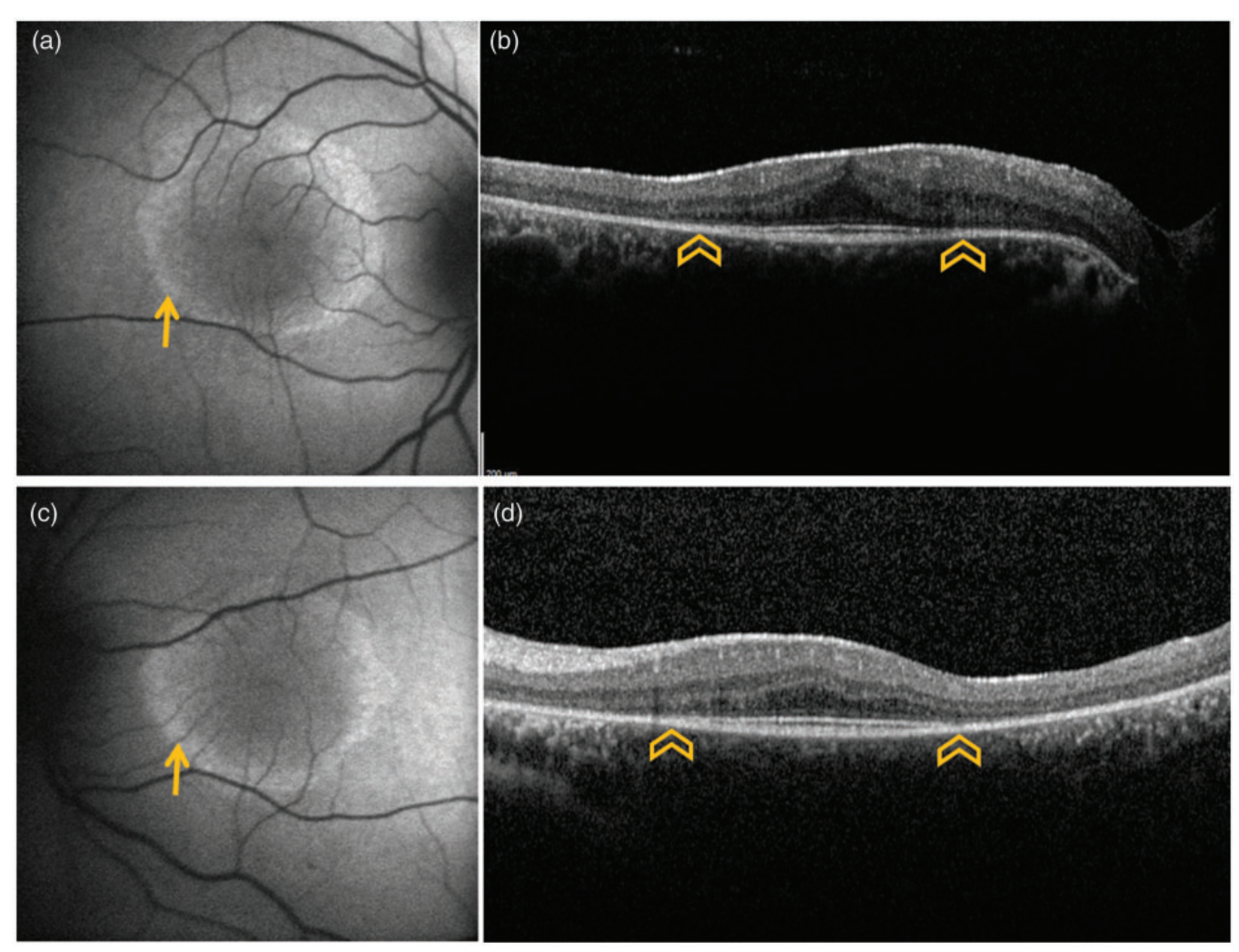

Figure 4 Fundus autofluorescence (a and c) and spectral-domain optical coherence tomography (b and d) of a 45-year-old female treated with hydroxychloroquine for 16 years. The arrows point out an area of increased autofluorescence in a bull's eye pattern. The arrow heads in the optical coherence tomography images delineate the paracentral thinning of the photoreceptors' layer.

Table 2 Comparison between SLE patients with and without hydroxychloroquine macular toxicity

\begin{tabular}{lccr}
\hline Variables & $\begin{array}{l}\text { With macular } \\
\text { toxicity }(\mathrm{n}=18)\end{array}$ & $\begin{array}{l}\text { Without macular } \\
\text { toxicity }(\mathrm{n}=143)\end{array}$ & p-value \\
\hline Age (years) & $54.9(11.2)$ & $46.7(13.4)$ & 0.014 \\
Male/female ratio & $1 / 9$ & $1 / 10.4$ & $0.860^{*}$ \\
HCQ cumulative dosage (g) & $2130.1(1341.6)$ & $939(893.5)$ & $<0.001$ \\
HCQ treatment time (years) & $14.7(9.6)$ & $6.9(6.4)$ & $<0.001$ \\
\hline
\end{tabular}

Results are expressed as mean (standard deviation), except for male/female ratio. ${ }^{*}$ Chi-square test, remaining p-values were obtained by Student's t-test.

$\mathrm{HCQ}=$ hydroxychloroquine

complication. Lupus retinopathy is a potentially sight-threatening complication that may manifest as retinal microangiopathy, retinal vasculitis or severe arterial or venous vaso-occlusion. ${ }^{2}$ Lupus choroidopathy is another sight-threatening manifestation that can occur in isolation or associated with lupus retinopathy. It may present with single or multiple areas of serous retinal detachment, retinal pigment epitheliopathy, retinal pigment epithelium (RPE) detachment, choroidal ischemia or effusion. $^{11,8}$ In our cohort, it was observed in
$0.6 \%$ of the patients, which also represents a slight decrease compared with previous studies. ${ }^{11,2}$ Moreover, lupus retinopathy and choroidopathy reflect high systemic disease activity and significantly correlate with central nervous system lupus and renal involvement, being a negative prognostic marker for survival. ${ }^{12,13}$ Over the last decade, significant improvements in the medical management of SLE have occurred, which have led to better systemic disease control as well as to a reduction in morbidity and mortality. The significant 
reduction in lupus retinopathy and choroidopathy is a direct consequence of the improvement in the standard of care of SLE.

Antimalarials, particularly HCQ, are nowadays the cornerstone of SLE medical therapy. Chronic medication with HCQ has proven beneficial in reducing lupus flares, end-organ damage and increasing survival rates in these patients. ${ }^{14,15}$ However, this benefit comes with a cost. Earlier studies reported an HCQ toxicity prevalence of $0-4 \%{ }^{16}$ However, the widespread use of these drugs for longer periods, with higher cumulative dosages and improvements in the screening strategy over recent years, have led to an increase in these estimates to $7.5-13.1 \% .{ }^{17,18}$ In our study, $11.2 \%$ of patients presented with HCQ macular toxicity, which is consistent with these later studies. The subgroup of patients with HCQ toxicity was a high-risk group, with a mean treatment time of approximately 15 years and a cumulative dosage of approximately $2130 \mathrm{~g}$. The pattern of HCQ retinal toxicity in clinical practice has also changed. In the past, the typical clinical picture was the classic bull's eye maculopathy with a paracentral ring scotoma, as a consequence of a paracentral ring of RPE atrophy. These defects were irreversible and often progressive, even after discontinuing the drug. ${ }^{19}$ Nowadays, modern screening methods, including SD-OCT, macular automated threshold visual fields, FAF, and mfERG, allow us to detect early paracentral photoreceptor damage, before the RPE affection takes place. ${ }^{6}$ Atypical patterns of maculopathy, such as cystoid macular degeneration or RPE detachment, like those observed in our cohort, have already been described. ${ }^{20,21}$

Another interesting finding in our study was the relative increase in the diagnosis of cataracts and glaucoma $(11.2 \%$ and $3.1 \%$ respectively). These numbers supersede those of earlier literature but are consistent with a more recent study, by Carli et al. that reported cataracts in $29 \%$ of patients and glaucoma in 3\% of cases. ${ }^{7,22}$ This tendency for an increase in the diagnosis of cataracts and glaucoma in SLE patients is related to chronic corticosteroid therapy, as there is an association between cumulative corticosteroid dosage and the incidence of these complications. ${ }^{22}$ Age is another important risk factor for both glaucoma and cataracts. Given the significant improvements in medical management of SLE, these patients tend to live longer and thus additionally suffer the effects of age-related ophthalmic diseases.

In conclusion, there has been a paradigm shift in ocular involvement in SLE patients. We observed a significant reduction in ophthalmic complications directly related to systemic disease activity, particularly lupus retinopathy. On the other hand, there has been an increase in drug and age-related ocular complications, such as HCQ maculopathy, cataracts and glaucoma. These phenomena are a consequence of improvements in the treatment of SLE and the associated increase in life expectancy of these patients. These results highlight the importance of regular ophthalmic screening, even in asymptomatic and systemically controlled SLE patients.

\section{Declaration of conflicting interests}

The authors declared no potential conflicts of interest with respect to the research, authorship, and/or publication of this article.

\section{Funding}

The authors disclosed receipt of the following financial support for the research, authorship, and/or publication of this article: This study was funded by the José de Mello Saúde - Hospital CUF Descobertas.

\section{ORCID iDs}

\section{A Dias-Santos (D) https://orcid.org/0000-0003- 3295-360X \\ M Francisca Moraes-Fontes (D) https://orcid.org/ 0000-0002-8917-6592}

\section{References}

1 Jensen J, Bergem H, Gilboe I, Husby G, Axéll T. Oral and ocular sicca symptoms and findings are prevalent in systemic lupus erythematosus. J Oral Pathol Med 1999; 28: 317-322.

2 Dias-Santos A, Proença RP, Tavares Ferreira J, et al. The role of ophthalmic imaging in central nervous system degeneration in systemic lupus erythematosus. Autoimmun Rev 2018; 17: 617-624.

3 Davies JB, Rao PK. Ocular manifestations of systemic lupus erythematosus. Curr Opin Ophthalmol 2008; 19: 512-518.

4 Tang C, Godfrey T, Stawell R, Nikpour M. Hydroxychloroquine in lupus: emerging evidence supporting multiple beneficial effects. Intern Med $J$ 2012; 42: 968-78.

5 Hochberg MC. Updating the American College of Rheumatology revised criteria for the classification of systemic lupus erythematosus. Arthritis Rheum 1997; 40: 1725.

6 Marmor MF, Kellner U, Lai TYY, Melles RB, Mieler WF. Recommendations on screening for chloroquine and hydroxychloroquine retinopathy (2016 revision). Ophthalmology 2016; 123: 1386-1394.

7 El-Shereef RR, Mohamed AS, Hamdy L. Ocular manifestation of systemic lupus erythematosus. Rheumatol Int 2013; 33: 1637-1642. 
8 Silpa-Archa S, Lee JJ, Foster CS. Ocular manifestations in systemic lupus erythematosus. Br J Ophthalmol 2016; 100: 135-141.

9 Pasoto SG, Martins VA de O, Bonfa E. Sjögren's syndrome and systemic lupus erythematosus: links and risks. Open Access Rheumatol Res Rev 2019; 29: 33-45.

10 Conigliaro P, Cesareo M, Chimenti MS, et al. Take a look at the eyes in Systemic Lupus Erythematosus: A novel point of view. Autoimmun Rev 2019; 247-254.

11 Nguyen QD, Uy HS, Akpek EK, Harper SL, Zacks DN, Foster CS. Choroidopathy of systemic lupus erythematosus. Lupus 2000; 9: 288-298.

12 Stafford-Brady FJ, Urowitz MB, Gladman DD, Easterbrook M. Lupus retinopathy. Patterns, associations, and prognosis. Arthritis Rheum 1988; 31: 1105-1110.

13 Jabs DA, Fine SL, Hochberg MC, Newman SA, Heiner GG, Stevens MB. Severe retinal vaso-occlusive disease in systemic lupus erythematosus. Arch Ophthalmol 1986; 104: 558-563.

14 Durcan L, Petri M. Immunomodulators in SLE: clinical evidence and immunologic actions. JAutoimmun 2016; 74: 73-84.

15 Shinjo SK, Bonfá E, Wojdyla D, et al. Antimalarial treatment may have a time-dependent effect on lupus survival: data from a multinational Latin American inception cohort. Arthritis Rheum 2010; 62: 855-862.
16 Marmor MF, Kellner U, Lai TYY, et al. Revised recommendations on screening for chloroquine and hydroxychloroquine retinopathy. Ophthalmology 2011; 118: 415-422.

17 Melles RB, Marmor MF. The risk of toxic retinopathy in patients on long-term hydroxychloroquine therapy. JAMA Ophthalmol 2014; 132: 1453-1460.

18 Palma Sánchez D, Rubio Velazquez E, Soro Marín S. Retinal toxicity due to antimalarials: frequency and risk factors. Reumatol Clin 2013; 9: 259-262.

19 Marmor MF, Hu J. Effect of disease stage on progression of hydroxychloroquine retinopathy. JAMA Ophthalmol 2014; 132: 1105-1112.

20 Kellner S, Weinitz S, Farmand G, Kellner U. Cystoid macular oedema and epiretinal membrane formation during progression of chloroquine retinopathy after drug cessation. $\mathrm{Br} J$ Ophthalmol 2014; 98: 200-206.

21 Lee WJ, Ko MK, Lee BR. Hydroxychloroquine retinopathy combined with retinal pigment epithelium detachment. Cutan Ocul Toxicol 2012; 31: 144-147.

22 Carli L, Tani C, Querci F, et al. Analysis of the prevalence of cataracts and glaucoma in systemic lupus erythematosus and evaluation of the rheumatologists' practice for the monitoring of glucocorticoid eye toxicity. Clin Rheumatol 2013; 32: 1071-1073. 
Copyright of Lupus is the property of Sage Publications Inc. and its content may not be copied or emailed to multiple sites or posted to a listserv without the copyright holder's express written permission. However, users may print, download, or email articles for individual use. 